\title{
AUTOMATIC TUNING OF QUALITY FACTORS FOR VHF CMOS FILTERS
}

\author{
Bram Nauta ${ }^{1}$ and Evert Seevinck ${ }^{2}$
}

1) University of Twente, dept. Electrical Engineering, IC- technology and Electronics Group, P.O. Box 217, 7500 AE Enschede, The Netherlands.

\section{$\underline{\text { Abstract }}$}

A novel circuit for tuning both the quality factors and the cut-off frequency of an integrated filter is described. The Q-tuning circuit itself has no signal carrying nodes and is therefore suitable for filters at very high frequencies [5]; up to $100 \mathrm{MHz}$.

\section{Introduction}

To correct the frequency response of an integrated filter for process and temperature variations, tuning of the cut-off frequency [1] (f-tuning) is generally applied. Several filters are also provided with automatic tuning of the quality factors (Q-tuning) [2]. Combined $\mathrm{f}$ - and Q-tuning can be applied with either a master VCF [2] (Voltage Controlled Filter). or a master VCO [3] (Voltage Controlled Oscillator).

In fig. 1 the method using a master VCO is illustrated. Consider first the Q-tuning loop. The Q-loop controls the amplitude of the VCO in such a way that it will oscillate with a constant amplitude. If the $\mathrm{Q}$ of the VCO is infinite, then the VCO will oscillate harmonically with a constant amplitude (the poles are exactly on the $\mathrm{j} \omega$ axis of the complex plane). If the voltage used for tuning the $Q$ of the two integrators in the master VCO is copied to the (matched) integrators
2) Philips Research Laboratories, P.O. Box 80.000, 5600 JA Eindhoven, The Netherlands in the slave filter, the quality factors of the filter will automatically be correct. The amplitude of the VCO signal is uncritical as long as the integrators in the VCO operate in their linear region.

The f-control loop is a well known phase locked loop (PLL) which locks the oscillating frequency to an external reference frequency. The voltage used for tuning the frequency of the VCO is copied to the slave filter.

The combination of $\mathrm{f}$ - and Q-tuning is possible if the $f-$ and Q-control loops are independent. This is difficult in practice. If the Q-tuning loop is much faster than the $\mathrm{f}$ - tuning loop, the f- tuning loop will be quasi static and then the $\mathrm{f}$ - and Q-loops become practically independent. For VHF filters the Q-loop must be fast enough to tune the VCO which oscillates at least at the cut-off frequency of the filter, which can be up to $110 \mathrm{MHz}$ [5]. The Q-tuning loop must therefore be very fast.

This paper describes a Q-tuning technique without a physical loop, so that it is very fast and therefore suitable for VHF.

\section{A VHF filter approach}

Recently, an approach has been introduced for VHF transconductance-C filters [4] which makes use of a

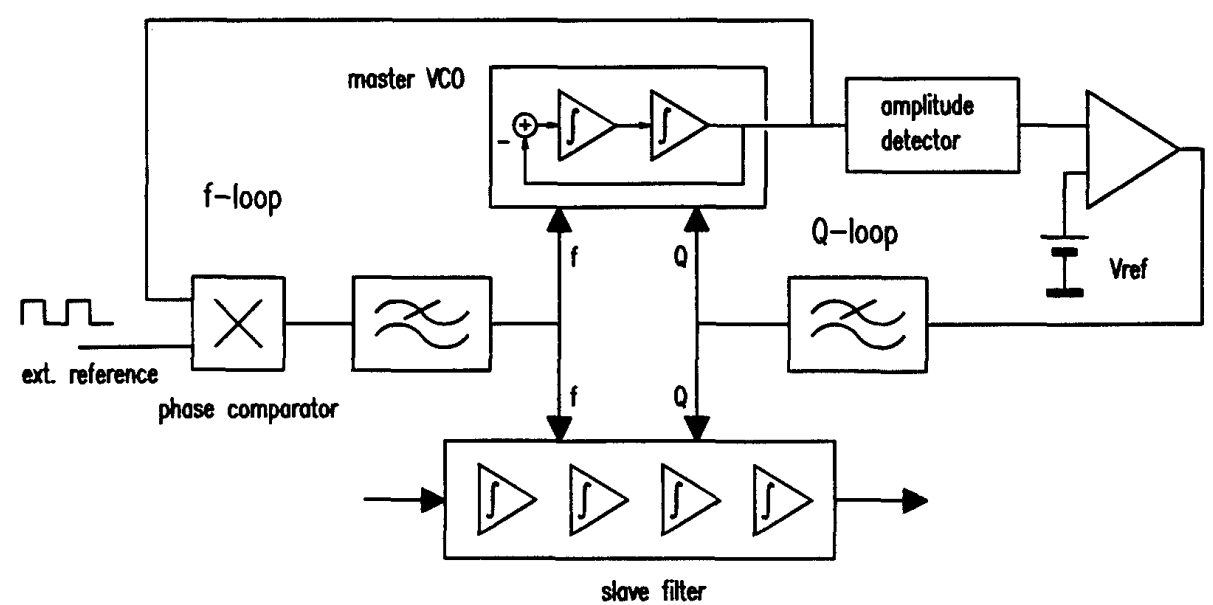

fig. 1 Combined $\mathrm{f}$ - and $\mathrm{Q}$ - tuning loops.

CH2868-8/90/0000-1147\$1.00 (c) 1990 IEEE 


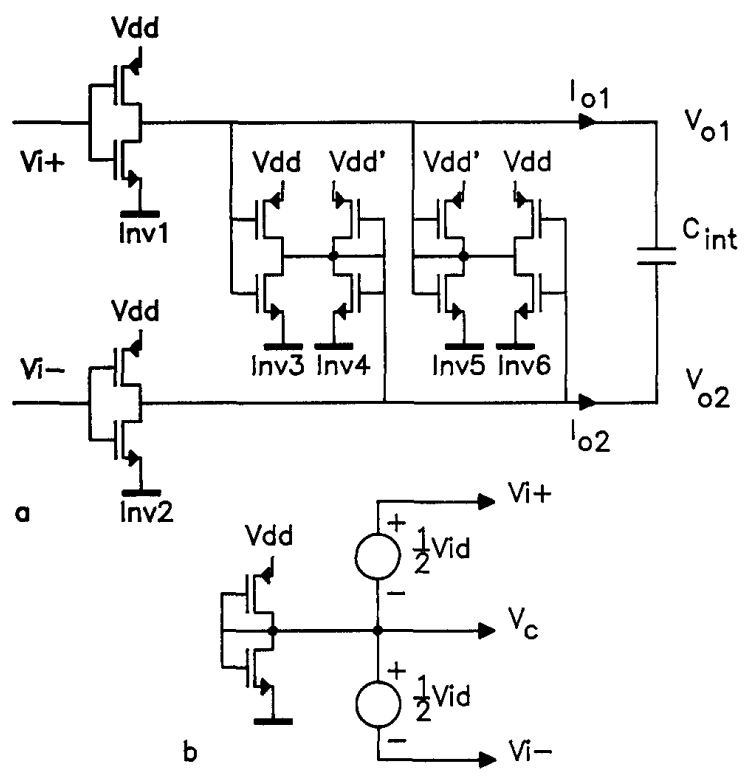

fig. $2 \mathrm{Li}$ ear VHF transconductance element [4].

transconductance element without internal nodes.

This transconductance element is given in figure 2a.

The basic V-I conversion is performed by two CMOS inverters Inv1 and Inv2. If these inverters are driven by a differential input voltage $V_{\text {id }}$, balanced around the common mode level $V_{C}$ ( see fig. $2 b$ ), the differential output current will be linear with the differential input voltage. This can be shown as follows.

If the drain currents of an N-channel and a P-channel MOS transistor in strong inversion and saturation are written as:

$I_{d n}=\frac{\beta_{n}}{2}\left(V_{g s}-V_{t n}\right)^{2}$ and $I_{d p}=\frac{\beta p}{2}\left(V_{g s}-V_{t p}\right)^{2}$

the differential output current of the two inverters can be written as:

$I_{o d}=I_{o 2}-I_{o 1}=v_{1 d}\left(v_{d d}-v_{t n}-\left|V_{t p}\right|\right) \sqrt{\beta_{n} \cdot \beta_{p}}=v_{i d} \cdot g m_{d}$

The transconductance $\mathrm{gm}_{\mathrm{d}}$ can be tuned by means of $\mathrm{V}_{\mathrm{dd}}$. The common mode level of the output voltages $V_{02}$ and $\mathrm{V}_{\mathrm{ol}}$ is controlled by the four inverters Inv3-Inv6. Inv4 and Inv5 are shunted as resistors $1 / \mathrm{gm}_{4}$ and $1 / \mathrm{gm}_{5}$. The output currents of Inv3 and Inv6, $\mathrm{gm}_{3}\left(\mathrm{~V}_{\mathrm{c}}-\mathrm{V}_{\mathrm{ol}}\right)$ and $\mathrm{gm}_{6}\left(\mathrm{~V}_{\mathrm{c}}-\mathrm{V}_{\mathrm{o} 2}\right)$ respectively, are injected into these resistors.

For common input signals the result is that the " $\mathrm{V}_{\text {o1 }}$ " node is loaded with a resistor $1 /\left(\mathrm{gm}_{5}+\mathrm{gm}_{6}\right)$ and the " $\mathrm{V}_{02}$ " node with a resistor $1 /\left(\mathrm{gm}_{3}+\mathrm{gm}_{4}\right)$. For differential signals the " $V_{01}$ node is loaded with a resistor $1 /\left(\mathrm{gm}_{5}-\mathrm{gm}_{6}\right)$ and the $" \mathrm{~V}_{02}$ " node is loaded with a resistor $1 /\left(\mathrm{gm}_{4}-\mathrm{gm}_{3}\right)$. If the inverters have the same supply voltage and geometry, all the gm's will ideally be equal. Thus the network Inv3-Inv6 forms a low-ohmic load for common signals and a high-ohmic load for differential signals, resulting in a controlled common mode voltage level of the outputs.

The DC-gain of the transconductor-C integrator is increased by loading the transconductor with a negative resistance for differential signals. By choosing $\mathrm{gm}_{3}>\mathrm{gm}_{4}, \mathrm{gm}_{5}=\mathrm{gm}_{4}$ and $\mathrm{gm}_{6}=\mathrm{gm}_{3}$ this negative resistance, $1 / \mathrm{\Delta gm}=1 /\left(\mathrm{gm}_{4}-\mathrm{gm}_{3}\right)=1 /\left(\mathrm{gm}_{5}-\mathrm{gm}_{6}\right)$, is simply implemented, without adding extra nodes to the circuit: The width of the transistors in Inv4 and Inv5 is designed slightly smaller than those of Inv3 and Inv6. In order to make $\mathrm{Q}$ tuning possible, the DC-gain of the integrators is fine-tuned during operation with a separate supply voltage $\mathrm{V}_{\mathrm{dd}}$ for Inv4 and Inv5. Increasing $V_{d d}$ with constant $V_{d d}$ results in a lower DC-gain of the integrator and therefore in lower quality factors of the filter. Thanks to the absence of internal nodes and the tunable output resistance, the phase error of the transconductance-C integrator will be very small up to very high frequencies.

With this transconductor filters at very high frequencies can be made. Figure 3 , for example, shows the measured frequency response of a $100 \mathrm{MHz}$ third order elliptic low-pass filter [5]. The f-tuning was done by means of $V_{d d}$ and $\mathrm{Q}$ tuning was done by means of $V_{\text {dd }}$. Both the $\mathrm{f}$ - and $\mathrm{Q}$ - tuning were performed manually. In the next section an automatic Q-tuning technique for the transconductance element of fig. 2 is presented.
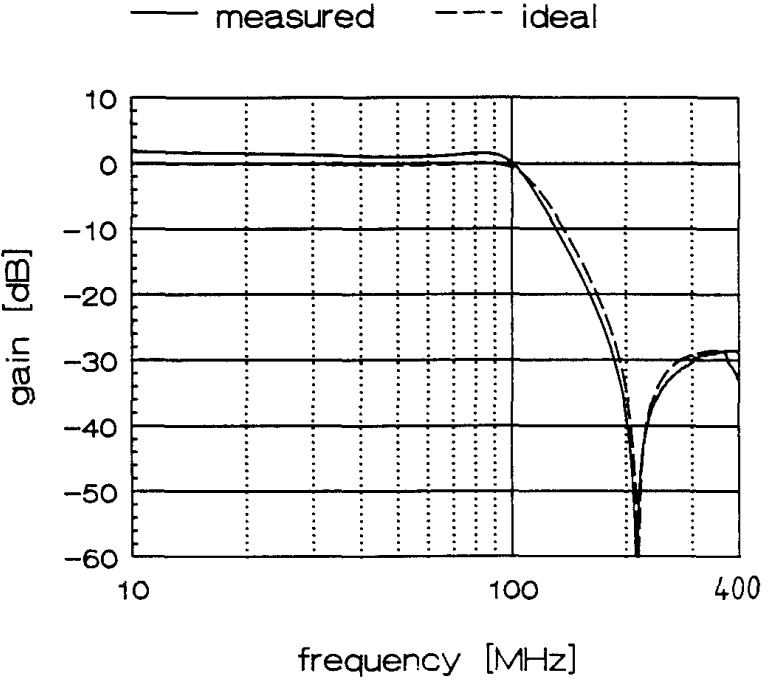

fig. 3 Frequency response of a $100 \mathrm{MHz}$ third order elliptic low-pass filter [5].

\section{Automatic Q-tuning}

With the transconductor described above the master VCO of fig. 4 can be made. If the $\mathrm{O}$ of the VCO is infinite, it will oscillate at a frequency determined by $V_{\text {dd }}$.

For every value of $V_{d d}$ there is only one value of $V_{d d}$, resulting in correct Q. The inverse is also true: for each $V_{d d}$ there is only one value of $V_{d d}$ so that the $Q$ is correct. It follows that the frequency can also be tuned with $V_{d d}$ ' if the Q-loop controls $V_{d d} . V_{d d}$ and $V_{d d}$ ' will then be related correctly.

This is very important for the Q-tuning circuit presented here.

Consider the VCO is oscillating harmonically with an amplitude $\mathrm{V}_{\mathrm{a}}$ at a frequency $\omega$. Using eq (1) the supply current $I_{d d}$ is calculated (see figure 4). This results in: 


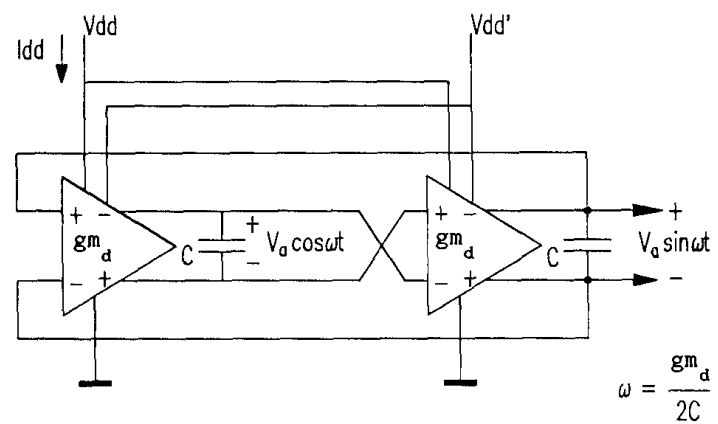

fig. 4 Voltage Controlled Oscillator.

$$
\begin{array}{r}
\mathbf{I}_{\mathrm{dd}}=2 \beta_{\mathrm{p}}\left[2\left[\frac{\sqrt{\beta_{\mathrm{n}} / \beta_{\mathrm{p}}}}{1+\sqrt{\beta_{\mathrm{n}} / \beta_{\mathrm{p}}}}\left(\mathrm{v}_{\mathrm{dd}}-\mathrm{v}_{\mathrm{tn}}-\left|\mathrm{v}_{\mathrm{tp}}\right|\right)\right]^{2}\right. \\
\left.+\frac{1}{4} \mathrm{v}_{\mathrm{a}}^{2}\left(\sin ^{2} \omega t+\cos ^{2} \omega t\right)\right)
\end{array}
$$

and since $\sin ^{2} \omega t+\cos ^{2} \omega t=1$, this can be written as:

$I_{d d}=2 \beta_{p}\left(2\left[\frac{\sqrt{\beta_{n}^{\prime} \beta_{p}}}{1+\sqrt{\beta_{n} / \beta_{p}}}\left(V_{d d}-V_{t n}-\left|V_{t p}\right|\right)\right]^{2}+\frac{1}{4} v_{d}^{2}\right]$

If $V_{a}=0$, therefore no oscillation, then $I_{d d}$ consists of the quiescent current of 8 inverters, biased in their linear region. In the case of oscillation, $V_{a} \neq 0$, the current is larger but remains constant. If the node $\mathrm{V}_{\mathrm{dd}}$ is supplied by means of a DC current source, with a value given by eq. (4), instead of a voltage source with value $V_{d d}$, the oscillator will oscillate with a constant and well controlled amplitude $V_{a}$. This can be seen as follows:

1) Suppose the amplitude $V_{a}$ tends to increase (poles of the $\mathrm{VCO}$ in the right half plane). With a constant $I_{d d}$ this implies from eq.(4) that $V_{d d}$ must decrease. With a (quasi static) constant $\mathrm{V}_{\mathrm{dd}}$ this implies that $\mathrm{gm}_{3}$, and $\mathrm{gm}_{6}$ ( fig. 2) decrease while $\mathrm{gm}_{4}$ and $\mathrm{gm}_{5}$ remain constant so that the oscillation is damped. The amplitude will therefore decrease.
2) Suppose the amplitude $V_{a}$ tends to decrease (poles in the left half plane). With a constant $I_{d d}$ this implies that $\mathrm{V}_{\mathrm{dd}}$ must increase. With a (quasi static) constant $V_{d d}$ ' this implies that $\mathrm{gm}_{3}$, and $\mathrm{gm}_{6}$ increase while $\mathrm{gm}_{4}$ and $\mathrm{gm}_{5}$ remain constant so that the oscillation is undamped. The amplitude will therefore increase.

The result of this mechanism is that, for a given $V_{d d}$, , $V_{d d}$ is controlled in such a way that the poles of the VCO will always be on the imaginary axis; the $Q$ factor of the VCO is then infinite.

If the resulting voltage $V_{d a}$ of the master $\mathrm{VCO}$ is copied to the filter by means of a buffer, the quality factors of the slave filter will automatically be correct.

The problem now is how to realize the current source $I_{d d}$ with the value given by eq. (4). This can be done as follows.

Usually $\dot{V}_{\mathrm{dd}} \approx \mathrm{V}_{\mathrm{dd}}$, with this in mind the current $\mathrm{I}_{\mathrm{dd}}$ can be made from $V_{d d}$, which in turn is determined by the frequency control loop. This is shown in figure 5 . The current $I_{0}$ is determined by $V_{d d}, V_{b}$, and the inverter parameters. The inverter in fig. 5 is equal to those in the VCO and all P-MOS and N-MOS transistors have equal geometries. The current $I_{0}$ can be calculated, this results in:

$I_{0}=\frac{1}{2} \beta_{p}\left[2\left[\frac{\sqrt{\beta_{n}^{\prime} \beta_{p}}}{1+\sqrt{\beta_{n}^{\prime} \beta_{p}}}\left(v_{d d}^{\prime}-v_{t n}-\left|v_{t p}\right|\right)\right]^{2}+2 v_{b}^{2}\right]$

Comparing eqs. (4) and (5) it can be seen that if:

$\mathrm{V}_{\mathrm{b}}=\frac{1}{4} \sqrt{2} \mathrm{~V}_{\mathrm{a}}$

the current $I_{0}$ has only to be multiplied with a factor four to obtain the current given by eq. (4), as long as $\mathrm{V}_{\mathrm{dd}} \approx \mathrm{V}_{\mathrm{dd}}$. This multiplication is simply performed with an 1:4 current mirror. The voltage buffer copies the voltage $V_{d d}$ to the slave filter. Normally the transistors deviate from ideal square law behavior, the result of this is that the current $I_{d d}$ of the VCO is not exactly constant. $I_{d d}$ will contain higher harmonics of the oscillation frequency $\omega$. The capacitance $C_{d d}$ however will drain these currents, so that the ripple in $V_{d d}$ remains very small. The capacitance $C_{d d}$ is the $\mathrm{N}$-well to substrate capacitance of the P-MOS transistors, which will be on chip. If necessary the buffer can in addition be preceded by a simple low pass filter.

Note, that if the circuit of fig. 5, the VCO and the

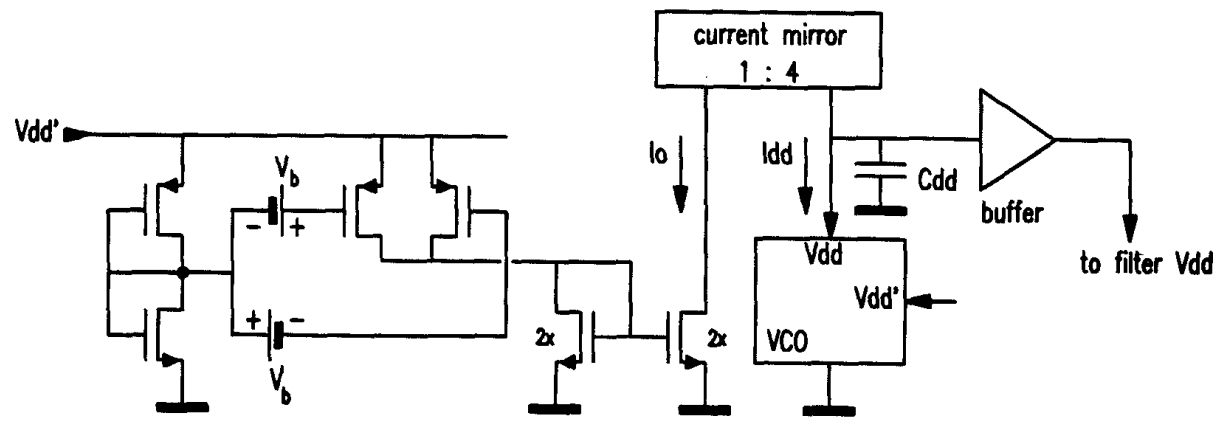

fig. 5 Circuit to generate the current $I_{d d}$. 
slave filter all have the same temperature, temperature effects are compensated.

The advantage of this Q-tuning circuit is that it needs no fast amplitude detectors or rectifiers, owing to the intrinsic wideband amplitude detection provided by the transconductance element of fig. 2 ( see also eq.(3) ).

The circuit of fig. 5 has no signal carrying nodes. All nodes have a (quasi static) DC voltage. For this reason the circuit is suitable for very high frequencies. The only signal carrying nodes are the outputs of the two integrators inside the VCO. Furthermore the circuit is extremely simple; it only consists of one - quasi static - current source, two current mirrors and a

buffer.

\section{Experimental results}

The circuit of figure 5 and the VCO have been realized on breadboard, using commercially available CA3600 CMOS arrays. The voltage $\mathrm{V}_{\mathrm{b}}$ was chosen $0.5 \mathrm{~V}$. Using eq. (6), the amplitude $\mathrm{V}_{a}$ of the VCO is expected to be $0.5^{*} 2 \sqrt{2}=1.4 \mathrm{~V}$.

The VCO oscillates at frequencies up to $7 \mathrm{MHz}$. The results are plotted in figure 6 . The voltage $V_{d d}$ varies with $V_{d d}$ in such a way that the VCO oscillates with a constant amplitude of almost $1.4 \mathrm{~V}$-as expected. The frequency varies almost linearly with $V_{d d}$ as predicted by eq. (2).

Simulations indicate that an on-chip realization of the circuit will be able to operate at very high frequencies; up to $100 \mathrm{MHz}$.

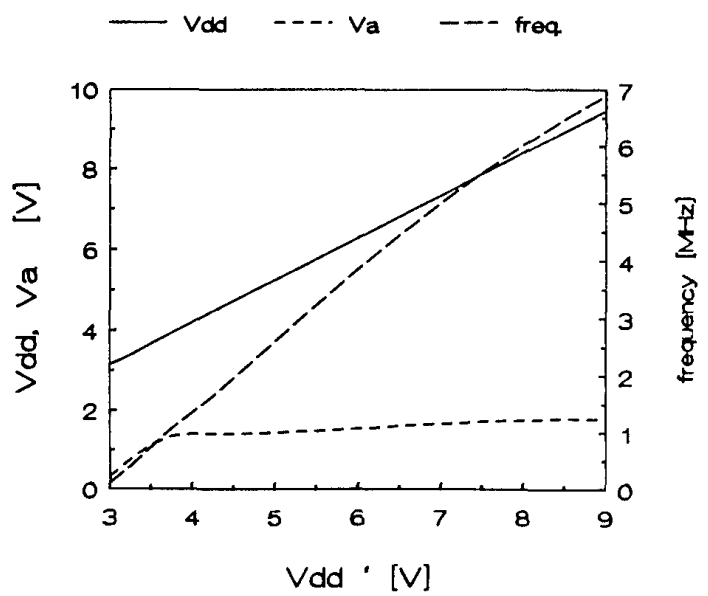

fig. 6 Experimental results obtained from a breadboard realization: $\mathrm{V}_{\mathrm{a}}$ (amplitude), $\mathrm{V}_{\mathrm{dd}}^{\mathrm{a}}$ and frequency of the VCO versus $V_{d d}$. The voltage $\mathrm{V}_{\mathrm{b}}$ was chosen $0.5 \mathrm{~V}$.

\section{Conclusions}

A VHF transconductance-C filter technique with automatic turing of the quality factors and the cut-off frequency, based on a VCO, was presented. The Q-tuning circuit is very simple and contains no signal carrying nodes resulting in a well contrclled $Q$ up to very high frequencies $(100 \mathrm{MHz})$. Experimental results of a breadboard realization of the Q-tuning circuit were presented, giving good results.

This work was sponsored by the Dutch IOP (Innovative Research Projects) program. The authors wish to thank W.J.A. de Heij, E. Klumperink, K. Hoen and C.T. Klein for fruitful discussions.

\section{References}

[1] K.Tan and P.R. Gray: "Fully Integrated Analog Filters Using Bipolar-JFET Technology" IEEE Journal of Solid State Circuits Vol. SC-13, No. 6, December 1978, pp 814-821.

[2] C. Chiou and R. Schaumann:"Design and Performance of a Fully Integrated Bipolar $10.7 \mathrm{MHz}$ Analog Bandpass Filter", IEEE Journal of Solid State Circuits, Vol. SC-21, No.1, February 1986, pp6-14.

[3] Y. Wang, F. Lu and A.A. Abidi: "A $12.5 \mathrm{MHz}$ CMOS Continuous Time Bandpass Filter", Digest of technical papers, ISSCC 1989.

[4] B. Nauta and E. Seevinck: "A linear Transconductance Element for VHF Filters", Electronics Letters, Vol.25, No.7, 30th March 1989, pp 448-450.

[5] B. Nauta and E. Seevinck: "A $110 \mathrm{MHz}$ CMOS Transconductance-C Low-Pass Filter", Digest of technical papers ESSCIRC '89 Vienna, Austria, September 1989. 